\title{
Synthesis and biological profile of substituted benzimidazoles
}

\author{
Neelam Vashist ${ }^{1}$, Surinder Singh Sambi², Balasubramanian Narasimhan ${ }^{3 *}$, Sanjiv Kumar ${ }^{3}$, Siong Meng Lim ${ }^{4,5}$, \\ Syed Adnan Ali Shah ${ }^{4,6}$, Kalavathy Ramasamy ${ }^{4,5}$ and Vasudevan Mani ${ }^{7}$
}

\begin{abstract}
Background: A series of benzimidazole derivatives was developed and its chemical scaffolds were authenticated by NMR, IR, elemental analyses and physicochemical properties. The synthesized compounds were screened for their antimicrobial and antiproliferative activities.

Results and discussion: The synthesized benzimidazole compounds were evaluated for their antimicrobial activity using the tube dilution method and were found to exhibit good antimicrobial potential against selected Gram negative and positive bacterial and fungal species. The compounds were also assessed for their anticancer activity exhibited using the SRB assay and were found to elicit antiproliferative activity against MCF7 breast cancer cell line, which was comparable to the standard drug.

Conclusion: Antimicrobial screening results indicated that compounds $\mathbf{1 , 2}$ and $\mathbf{1 9}$ to be promising antimicrobial agents against selected microbial species and comparable to standard drugs which included norfloxacin and fluconazole. The anticancer screening results revealed that compounds, 12, 21, $\mathbf{2 2}$ and $\mathbf{2 9}$ to show the highest activity against MCF7 and their $\mathrm{IC}_{50}$ values were more potent than 5 -fluorouracil.
\end{abstract}

Keywords: Benzimidazoles, Synthesis, Antimicrobial activity, Anticancer activity

\section{Background}

The emergence of antibiotic-resistant microorganisms such as fluoroquinolone-resistant Escherichia coli, Streptococcus pneumonia, carbapenem-resistant Klebsiella pneumonia, vancomycin-resistant enterococci and methicillin-resistant Staphylococcus aureus is becoming a serious health issue worldwide. There is a critical need to develop new chemotherapeutic agents with different mechanism of action [1].

Cancer is a deadly disease prevalent in both the developing as well as the developed countries. In spite of significant improvements in recognition and treatment of cancer, the incidence of certain types of malignancy is still on the rise. Current treatments such as cytotoxic chemotherapy and radiotherapy yielded only transient therapeutic aids that are accompanied by severe adverse

\footnotetext{
*Correspondence: naru2000us@yahoo.com

${ }^{3}$ Faculty of Pharmaceutical Sciences, Maharshi Dayanand University, Rohtak 124001, India

Full list of author information is available at the end of the article
}

effects. This is due to their toxic effects against normal growing cells. Concerted effort is, therefore, required to eliminate or at least reduce these incidences significantly [2].

Recent findings suggest that substituted benzimidazole derivatives possess potential chemotherapeutic activity with reduced toxic effects. Antibacterial activity of substituted benzimidazole derivatives can be explained by their competition with purines, an integral part of bacterial strain, resulting in inhibition of bacterial nucleic acids and proteins synthesis [3]. Compounds containing benzimidazole moiety such as thiabendazole, parbendazole, mebendazole, albendazole, cambendazole and flubendazole had also been reported for their antihelminthic activity. Similarly, the proton pump inhibitors, omeprazole, lansoprazole, rabeprazole, pantoprazole, had been reported for their use in the management of acid related disorders. In fact, benzimidazole derivatives had found their applications as antioxidant [4], antimicrobial [5], antihelmintic

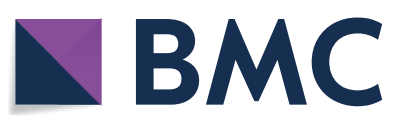

(c) The Author(s) 2018. This article is distributed under the terms of the Creative Commons Attribution 4.0 International License (http://creativecommons.org/licenses/by/4.0/), which permits unrestricted use, distribution, and reproduction in any medium, provided you give appropriate credit to the original author(s) and the source, provide a link to the Creative Commons license, and indicate if changes were made. The Creative Commons Public Domain Dedication waiver (http://creativecommons.org/ publicdomain/zero/1.0/) applies to the data made available in this article, unless otherwise stated. 
[6], anticancer [7], antiviral [8], antiallergic [9], antiarthritic [10] and anti-mycobacterial agents [11].

In light of above, the present study was undertaken to synthesise and evaluate the antimicrobial and anticancer potentials of substituted benzimidazole derivatives.

\section{Results and discussion \\ Chemistry}

Target compounds (1-30) were synthesized by following procedure outlined in Scheme 1. The physicochemical data of the target compounds are presented in Table 1. The synthesized compounds were evaluated on the basis of spectral analysis: IR, NMR and Mass and elemental analyses which were in full agreement with their proposed molecular structures. The formation of Schiff bases is confirmed by the presence of $\mathrm{N}=\mathrm{CH}$ str., at around $1560 \mathrm{~cm}^{-1}$ in the IR spectra of synthesized compounds (1-11). Asym str., at around $1550 \mathrm{~cm}^{-1}$ indicated the presence of aromatic nitro group in 5,7 , 13, 14, 16, 21-28 compounds. The presence of $\mathrm{C}-\mathrm{O}-\mathrm{C}$ str., of aralkyl showed methoxy group in $3, \mathbf{9}, \mathbf{1 1}, \mathbf{1 3}$, 17-20, 27 compounds. The $\mathrm{C}-\mathrm{H}$ str., at $1727 \mathrm{~cm}^{-1}$ confirmed the aliphatic aldehyde group in 10, 14 and 15 compounds. Furthermore, the appearance of $\mathrm{C}=\mathrm{O}$ str., at $1660 \mathrm{~cm}^{-1}$ and the absence of NH str., of imidazole at $3400 \mathrm{~cm}^{-1}$ confirmed the synthesis of methanone derivatives (12-30). The multiplet corresponds to 6.697$7.823 \delta$ ppm confirmed the presence of aromatic protons of aryl nucleus and benzimidazole. The appearance of singlet at around $9.580 \delta \mathrm{ppm}$ confirmed the Schiff bases $(\mathrm{N}=\mathrm{CH}-)$. The singlet peak at $3.426 \delta \mathrm{ppm}$ indicated the presence of dimethyl group in compounds, $\mathbf{1 , 6 , 2 2}$, 29 and 30. The doublet peak observed at 1.273-1.276 $\delta$ ppm which confirmed the presence of aliphatic methyl group in the synthesized compounds, 8,12 and 15 . The multiplet showed at $1.243-2.496 \delta \mathrm{ppm}$ confirmed the presence of $\mathrm{CH}_{2}$ chain of palmitoyl group in the structure of compounds (20, 28 and 29). Further confirmation was made on the basis of ${ }^{13} \mathrm{C}-\mathrm{NMR}$ and MS spectral analyses. The results of $\mathrm{C}, \mathrm{H}, \mathrm{N}$ analysis are within limits of $\pm 0.3 \%$.

\section{Anticancer activity}

The synthesized benzimidazole derivatives were screened for their anticancer activity against MCF7 (ATCC HTB-22), an oestrogen receptor positive human breast adeno-carcinoma cell line. Anticancer screening results (Table 2) indicated that compound $22\left(\mathrm{IC}_{50}=0.9 \mu \mathrm{M}\right)$ was found to be the most potent when compared to the standard drug, 5-fluorouracil $\left(\mathrm{IC}_{50}=35.4 \mu \mathrm{M}\right)$. Other compounds which included 12, 21 and 29 also exhibited more potent antiproliferative results $\left(\mathrm{IC}_{50}=7.0,5.4\right.$ and $5.5 \mu \mathrm{M}$, respectively) when compared to the standard drug. These compounds may be used as drug leads for discovery of new anticancer agents.

\section{Antimicrobial activity}

Antimicrobial activity results (Table 3 ) indicated that the compounds possessed good antimicrobial activity against the tested bacterial and fungal strains. Compound 1 showed good antibacterial activity against $E$. coli $\left(\mathrm{MIC}_{e c}=5.4 \mu \mathrm{M}\right)$ and B. subtilis $\left(\mathrm{MIC}_{b s}=10.7 \mu \mathrm{M}\right)$, whereas compound 19 was found to be more potent against $S$. aureus $\left(\mathrm{MIC}_{s a}=12.4 \mu \mathrm{M}\right)$. The reference drug, norfloxacin, yielded MIC of $4.7 \mu \mathrm{M}$ against the tested microorganisms. The antifungal activity results indicated that compound $\mathbf{2}$ showed good activity against $C$. albicans $\left(\mathrm{MIC}_{c a}=5.4 \mu \mathrm{M}\right.$. Compound 19, on the other hand, was the most potent antifungal agent against $A$. niger $\left(\mathrm{MIC}_{a n}=3.1 \mu \mathrm{M}\right)$ in comparison to fluconazole $(\mathrm{MIC}=5.0 \mu \mathrm{M})$, the reference drug. Thus, compound 19 may serve as a potential lead compound for the design of novel antifungal agents.

\section{Structure activity relationship}

The following structure activity relationship may be drawn from the antimicrobial and anticancer activities of the benzimidazole derivatives (Fig. 1):

- It has been noticed that the antibacterial activity of Schiff bases against $E$. coli enhanced due to the presence of vinyl group between benzimidazole amine and $\mathrm{N}$-benzylidene moiety and the substitution of electron releasing group at phenyl nucleus as in the compound 1 and the same moiety improved anticancer activity of methanone derivatives as in compound 22.

- The electron donating group placed at phenyl ring attached to $\mathrm{N}$-alylidene/arylidene moiety along with presence of electron withdrawing group on phenyl ring attached to methanone moiety improved antibacterial and antifungal activity of synthesized benzimidazole derivatives against bacterial and fungal strains as in compound 19.

\section{Experimental}

\section{Materials and methods}

All the laboratory reagents were procured from Sigma Aldrich and were used without any purification. Melting points were determined on Sonar melting point apparatus in an open capillary tube and are uncorrected. Purity of the compound was ascertained by commercialized (E-Merck Kieselgel 60 F254) TLC plates. The Infrared spectrum was recorded in $\mathrm{KBr}$ discs on a ShimadzuFTIR 8400 S spectrometer $\left(v_{\max }\right.$ in $\left.\mathrm{cm}^{-1}\right)$. Proton and 


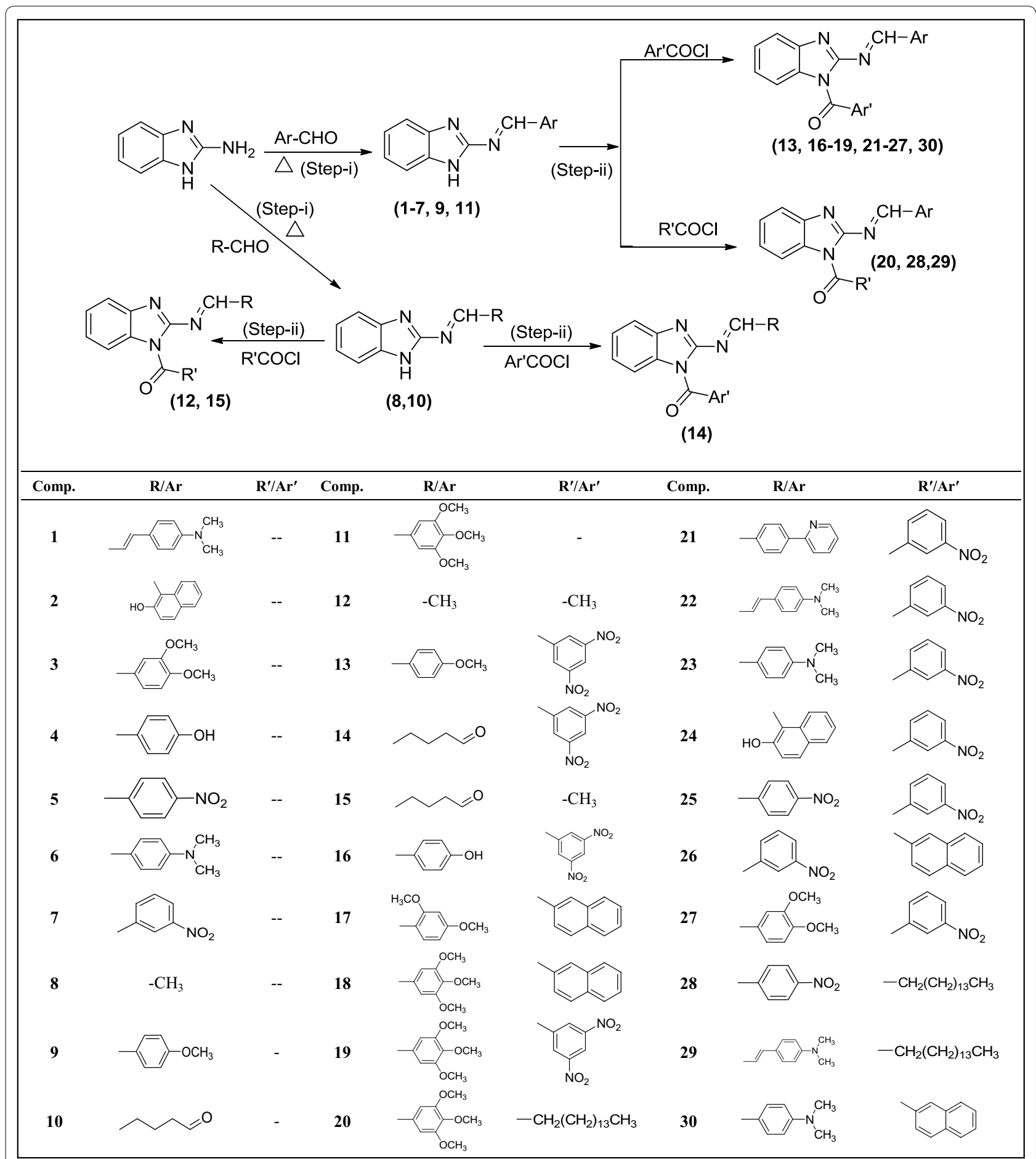

Scheme 1 Synthesis of benzimidazole derivatives (1-30). Reaction condition: Step i: 2-Aminobenzimidazole, substituted aldehyde, ethanol, glacial acetic acid, reflux for 4-5 h (RT), Step ii: Schiff's base, different acylchlorides, dimethylformamide, triethylamine, stir for 24 h (RT) 
Table 1 Physicochemical characteristic of the synthesized compounds

\section{Comp. Molecular structures with stereochemistry \\ 1<smiles>CN(C)c1ccc(/C=C/C=[N+]=Nc2nc3ccccc3[nH]2)cc1</smiles>

(E)-N-((E)-3-(4-(Dimethylamino)phenyl) allylidene)$1 \mathrm{H}$-benzo[d]imidazol-2-amine

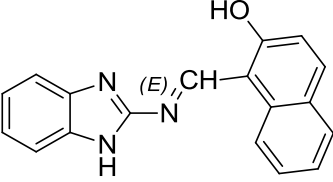

(E)-1-(((1H-Benzo[d]imidazol-2-yl)imino)methyl) naphthalen-2-ol<smiles>COc1ccc(C=Nc2nc3ccccc3[nH]2)cc1OC</smiles>

(E)-N-(3,4-Dimethoxybenzylidene)-1H-benzo[d] imidazol-2-amine<smiles>Oc1ccc(C=Nc2nc3ccccc3[nH]2)cc1</smiles>

(E)-4-(((1H-Benzo[d]imidazol-2-yl)imino)methyl) phenol<smiles>O=[N+]([O-])c1ccc(C=Nc2nc3ccccc3[nH]2)cc1</smiles>

(E)-N-(4-Nitrobenzylidene)-1H-benzo[d]imidazol2-amine

6<smiles>CN(C)c1ccc(C=Nc2nc3ccccc3[nH]2)cc1</smiles>

(E)-N-(4-(Dimethylamino)benzylidene)-1H-benzo[d] imidazol-2-amine<smiles></smiles>

(E)-N-(3-Nitrobenzylidene)-1H-benzo[d]imidazol2-amine<smiles>Cc1nc2ccccc2[nH]1</smiles>

(E)-N-Ethylidene-1H-benzo [d]imidazol-2-amine<smiles>COc1ccc(C=Nc2nc3ccccc3[nH]2)cc1</smiles>

(E)-N-(4-Methoxybenzylidene)-1H-benzo[d] imidazol-2-amine

M. formula and $\mathrm{CHN}$ analyses

M. wt. Rf value \% Yield M.Pt. $\left({ }^{\circ} \mathrm{C}\right)$

$\mathrm{C}_{18} \mathrm{H}_{18} \mathrm{~N}_{4}$ : Anal calcd: $\mathrm{C}, 74.46 ; \mathrm{H}, 6.25 ; \mathrm{N}, 19.30$;

Found: $\mathrm{C}, 74.43 ; \mathrm{H}, 6.27 ; \mathrm{N}, 19.33$

$290.40 \quad 0.76^{\mathrm{a}}$

76

$228-230$

$\mathrm{C}_{18} \mathrm{H}_{13} \mathrm{~N}_{3} \mathrm{O}$ : Anal calcd: C, 75.25; $\mathrm{H}, 4.56 ; \mathrm{N}, 14.63$;

Found: $\mathrm{C}, 75.27 ; \mathrm{H}, 4.59 ; \mathrm{N}, 14.60$

$287.34 \quad 0.79^{\mathrm{a}} \quad 74$

$255-257$

$\mathrm{C}_{16} \mathrm{H}_{15} \mathrm{~N}_{3} \mathrm{O}_{2}$ : Anal calcd: $\mathrm{C}, 68.31 ; \mathrm{H}, 5.37 ; \mathrm{N}, 14.94$; Found: $\mathrm{C}, 68.34 ; \mathrm{H}, 5.35 ; \mathrm{N}, 14.97$

$\mathrm{C}_{14} \mathrm{H}_{11} \mathrm{~N}_{3} \mathrm{O}$ : Anal calcd: $\mathrm{C}, 70.87 ; \mathrm{H}, 4.67 ; \mathrm{Cl}, 17.71 ;$ Found: $\mathrm{C}, 70.88 ; \mathrm{H}, 4.65 ; \mathrm{Cl}, 17.73$

$237.28 \quad 0.75^{\circ}$

67

$220-222$

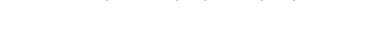

$\mathrm{C}_{14} \mathrm{H}_{10} \mathrm{~N}_{4} \mathrm{O}_{2}$ : Anal calcd: $\mathrm{C}, 63.15 ; \mathrm{H}, 3.79 ; \mathrm{N}, 21.04$ Found: $\mathrm{C}, 63.13 ; \mathrm{H}, 3.77 ; \mathrm{N}, 21.07$

$\mathrm{C}_{16} \mathrm{H}_{16} \mathrm{~N}_{4}$ : Anal calcd: $\mathrm{C}, 72.70 ; \mathrm{H}, 6.10 ; \mathrm{N}, 21.20$; Found: $\mathrm{C}, 72.73 ; \mathrm{H}, 6.12 ; \mathrm{N}, 21.22$

$264.360 .79^{a}$

82

$238-240$
$\mathrm{C}_{14} \mathrm{H}_{10} \mathrm{~N}_{4} \mathrm{O}_{2}$ : Anal calcd: $\mathrm{C}, 63.15 ; \mathrm{H}, 3.79 ; \mathrm{N}, 21.04 ; 266.28 \quad 0.76^{\mathrm{a}} \quad 76 \quad 190-192$ Found: $\mathrm{C}, 63.18 ; \mathrm{H}, 3.77 ; \mathrm{N}, 21.05$
$\mathrm{C}_{9} \mathrm{H}_{9} \mathrm{~N}_{3}$ : Anal calcd: C, 67.90; H, 5.70; N, 26.40; Found: $\mathrm{C}, 67.88 ; \mathrm{H}, 5.72 ; \mathrm{N}, 26.42$


Table 1 (continued)

\section{Comp. Molecular structures with stereochemistry}

10<smiles>O=CCCCC=Nc1nc2ccccc2[nH]1</smiles>

(E)-5-((1H-Benzo[d]imidazol-2-yl)imino)pentanal

11<smiles></smiles>

(E)-N-(3,4,5-Trimethoxybenzylidene)-1H-benzo[d] imidazol-2-amine

12<smiles>CC(=O)n1c(N=C(C)C)nc2ccccc21</smiles>

(E)-1-(2-(Ethylideneamino)-1H-benzo[d]imidazol$1-y \mid)$ ethanone

13<smiles>[N-]=[N+]([O-])c1cc(C(=O)n2c([N+](=O)[O-])nc3ccccc32)cc([N+](=O)[O-])c1</smiles>

(E)-(3,5-Dinitrophenyl) (2-((4-methoxy-benzylidene) amino)-1H-benzo[d]imidazol-1-yl)methanone

14<smiles>[C-]=Nc1nc2ccccc2n1C(=O)c1cc([N+](=O)[O-])cc([N+](=O)[O-])c1</smiles>

(E)-5-((1-(3,5-Dinitrobenzoyl)-1H-benzo[d]imidazol2-yl)imino)pentanal

15<smiles>[CH-]=C(CCCC=O)Nc1nc2ccccc2n1C(C)=O</smiles>

(E)-5-((1-Acetyl-1H-benzo[d]imidazol-2-yl)imino) pentanal

16<smiles>O=C(c1cc([N+](=O)[O-])cc([N+](=O)[O-])c1)n1c(N=Cc2ccc(O)cc2)nc2ccccc21</smiles>

(E)-(3,5-Dinitrophenyl)(2-((4-hydroxybenzylidene) amino)-1H-benzo[d]imidazol-1-yl)methanone

17

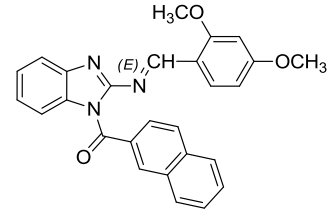

(E)-(2-((2,4-Dimethoxybenzylidene)amino)

-1H-benzo[d]imidazol-1-yl)(naphthalen-2-yl) methanone

\section{M. formula and $\mathrm{CHN}$ analyses}

M. wt. Rf value \% Yield M. Pt. $\left({ }^{\circ} \mathrm{C}\right)$

$\mathrm{C}_{12} \mathrm{H}_{13} \mathrm{~N}_{3} \mathrm{O}$ : Anal calcd: $\mathrm{C}, 66.96 ; \mathrm{H}, 6.09 ; \mathrm{N}, 19.52 ; \quad 215.28 \quad 0.75^{\mathrm{a}} \quad 78$ Found: $\mathrm{C}, 66.94 ; \mathrm{H}, 6.11 ; \mathrm{N}, 19.55$

$\mathrm{C}_{17} \mathrm{H}_{17} \mathrm{~N}_{3} \mathrm{O}_{3}$ : Anal calcd: $\mathrm{C}, 65.58 ; \mathrm{H}, 5.50 ; \mathrm{N}, 13.50 ; \quad 311.37 \quad 0.72^{\mathrm{a}}$ Found: $\mathrm{C}, 65.61 ; \mathrm{H}, 5.53 ; \mathrm{N}, 13.52$

\section{$\mathrm{C}_{11} \mathrm{H}_{11} \mathrm{~N}_{3} \mathrm{O}$ : Anal calcd: C, 65.66; $\mathrm{H}, 5.51 ; \mathrm{N}, 20.88$;}

$201.220 .63^{b}$

74

$262-265$ Found: C, 65.65; H, 5.54; N, 20.86

$\mathrm{C}_{22} \mathrm{H}_{15} \mathrm{~N}_{5} \mathrm{O}_{6}$ : Anal calcd: C, 59.33; $\mathrm{H}, 3.39 ; \mathrm{N}, 15.72 ; 445.38 \quad 0.58^{\mathrm{b}} \quad 68$ $243-245$ Found: $\mathrm{C}, 59.35 ; \mathrm{H}, 3.42 ; \mathrm{N}, 15.75$

$\mathrm{C}_{14} \mathrm{H}_{11} \mathrm{~N}_{3} \mathrm{O}_{2}$ : Anal calcd: $\mathrm{C}, 55.75 ; \mathrm{H}, 3.69 ; \mathrm{N}, 17.11 ; 253.26 \quad 0.66^{\mathrm{b}} \quad 65$ $162-164$ Found: $\mathrm{C}, 55.78 ; \mathrm{H}, 3.71 ; \mathrm{N}, 17.14$

$\mathrm{C}_{14} \mathrm{H}_{15} \mathrm{~N}_{3} \mathrm{O}_{2}$ : Anal calcd: C, 65.35; $\mathrm{H}, 5.88 ; \mathrm{N}, 16.33 ; \quad 257.290 .62^{\mathrm{b}} 7$ Found: $\mathrm{C}, 65.37 ; \mathrm{H}, 5.90 ; \mathrm{N}, 16.36$

$226-228$

$\mathrm{C}_{21} \mathrm{H}_{13} \mathrm{~N}_{5} \mathrm{O}_{6}$ : Anal calcd: $\mathrm{C}, 58.47 ; \mathrm{H}, 3.04 ; \mathrm{N}, 16.24 ; 431.360 .64^{\mathrm{b}} 70$ $175-177$ Found: C, 58.49; H, 3.05; N, 16.25

$435.470 .54^{b}$ 67 
Table 1 (continued)

Comp. Molecular structures with stereochemistry

18<smiles>C=Nc1nc2ccccc2n1C(=O)c1ccc2ccccc2c1</smiles>

(E)-Naphthalen-2-yl(2-((3,4,5-trimethoxybenzylidene)amino)-1H-benzo[d]imidazol-1-yl) methanone

19<smiles>[CH2-][C@H](c1cc(OC)c(OC)c(OC)c1)n1c([N+](=O)[O-])nc2ccccc21</smiles>

(E)-(3,5-Dinitrophenyl) (2-((3,4,5-trimethoxybenzylidene)amino)-1H-benzo[d]imidazol-1-yl) methanone

20

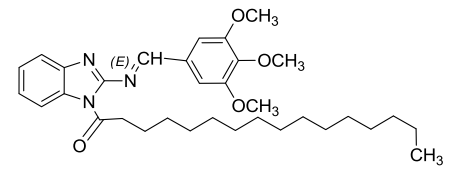

(E)-1-(2-((3,4,5-trimethoxybenzylidene)amino)- $1 \mathrm{H}$ benzo[d]imidazol-1-yl)hexadecan-1-one

21

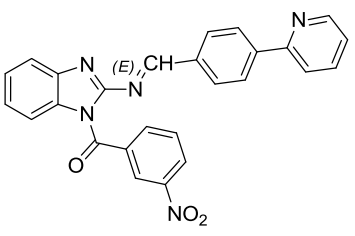

(E)-(3-Nitrophenyl)(2-((4-(pyridin-2-yl)benzylidene) amino)-1H-benzo[d]imidazol-1-yl)methanone

22

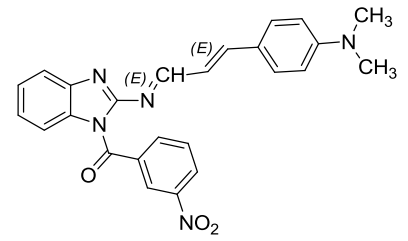

(2-((E)-((E)-3-(4-(Dimethylamino)phenyl) allylidene) amino)-1H-benzo[d]imidazol-1-yl)(3-nitrophenyl) methanone

23

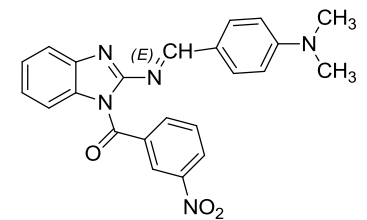

(E)-(2-((4-(Dimethylamino)benzylidene) amino)-1 $\mathrm{H}$ benzo[d]imidazol-1-yl)(3-nitrophenyl)methanone

\section{M. formula and $\mathrm{CHN}$ analyses}

M. wt. Rfvalue \% Yield M.Pt. $\left({ }^{\circ} \mathrm{C}\right)$

$\mathrm{C}_{28} \mathrm{H}_{23} \mathrm{~N}_{3} \mathrm{O}_{4}$ : Anal calcd: C, 72.24; $\mathrm{H}, 4.98 ; \mathrm{N}, 9.03$;

$465.50 .65^{b}$

75

$210-212$

Found: $\mathrm{C}, 72.27 ; \mathrm{H}, 4.95 ; \mathrm{N}, 9.05$ $\mathrm{C}_{24} \mathrm{H}_{19} \mathrm{~N}_{5} \mathrm{O}_{8}$ : Anal calcd: $\mathrm{C}, 57.03 ; \mathrm{H}, 3.79 ; \mathrm{N}, 13.86 ; 505.44 \quad 0.66^{\mathrm{b}} \quad 66 \quad$ 141-143
Found: $\mathrm{C}, 57.07 ; \mathrm{H}, 3.76 ; \mathrm{N}, 13.88$

$\mathrm{C}_{33} \mathrm{H}_{47} \mathrm{~N}_{3} \mathrm{O}_{4}:$ Anal calcd: $\mathrm{C}, 72.10 ; \mathrm{H}, 8.62 ; \mathrm{N}, 7.64 ; \quad 549.740 .62^{\mathrm{b}} \quad 78 \quad 136-138$
Found: $\mathrm{C}$. $72.11 ; \mathrm{H}, 8.65 ; \mathrm{N}, 7.67$

$\mathrm{C}_{26} \mathrm{H}_{17} \mathrm{~N}_{5} \mathrm{O}_{3}$ : Anal calcd: C, 69.79; $\mathrm{H}, 3.83 ; \mathrm{N}, 15.65 ; 447.44 \quad 0.57^{\mathrm{b}} \quad 67 \quad 142-144$ Found: $\mathrm{C}, 69.77 ; \mathrm{H}, 3.86 ; \mathrm{N}, 15.68$ $\mathrm{C}_{25} \mathrm{H}_{21} \mathrm{~N}_{5} \mathrm{O}_{3}:$ Anal calcd: $\mathrm{C}, 68.33 ; \mathrm{H}, 4.82 ; \mathrm{N}, 15.94 ; 439.47 \quad 0.59^{\mathrm{b}} \quad 82$
$\quad$ Found: $\mathrm{C}, 68.37 ; \mathrm{H}, 4.80 ; \mathrm{N}, 15.97$

$\mathrm{C}_{23} \mathrm{H}_{19} \mathrm{~N}_{5} \mathrm{O}_{3}$ : Anal calcd: $\mathrm{C}, 66.82 ; \mathrm{H}, 4.63 ; \mathrm{N}, 16.94 ; 413.430 .63^{\mathrm{b}} 76$

$131-133$ 
Table 1 (continued)

\section{Comp. Molecular structures with stereochemistry}

24<smiles>[C-]=Nc1nc2ccccc2n1C(=O)c1cccc([N+](=O)[O-])c1</smiles>

(E)-(2-(((2-hydroxynaphthalen-1-yl)-methylene) amino)-1H-benzo[d]imidazol-1-yl)(3-nitrophenyl) methanone

25<smiles>O=C(c1cccc([N+](=O)[O-])c1)n1c(N=Cc2ccc([N+](=O)[O-])cc2)nc2ccccc21</smiles>

(E)-(2-((4-Nitrobenzylidene)amino)-1H-benzo[d] imidazol-1-yl)(3-nitrophenyl) methanone

26<smiles>O=C(c1ccc2ccccc2c1)n1c(N=Cc2ccc([N+](=O)[O-])cc2)nc2ccccc21</smiles>

(E)-Naphthalen-2-yl(2-((4-nitrobenzylidene)amino)$1 \mathrm{H}$-benzo[d]imidazol-1-yl)methanone

27<smiles>[N-]=Cc1nc2ccccc2n1C(=O)c1cccc([N+](=O)[O-])c1</smiles>

(E)-(2-((3,4-Dimethoxybenzylidene)amino) -1H-benzo[d]imidazol-1-yl)(3-nitro phenyl) methanone

28<smiles>CCCCCCCCCCCCCCCC(=O)n1c(N=Cc2ccc([N+](=O)[O-])cc2)nc2ccccc21</smiles>

(E)-1-(2-((4-Nitrobenzylidene)amino)-1H-benzo[d] imidazol-1-yl)hexadecan-1-one

29<smiles>CCCCCCCCCCCCCCCC(=O)n1c([N-]C=Cc2ccc(N(C)C)cc2)nc2ccccc21</smiles>

(E)-1-(2-((4-(Dimethylamino)benzylidene) amino)1H-benzo[d]imidazol-1-yl)hexadecan-1-one

30<smiles>CN(C)c1ccc(/C=N\c2nc3ccccc3n2C(=O)c2ccc3ccccc3c2)cc1</smiles>

(E)-(2-((4-(Dimethylamino)benzylidene) amino)$1 \mathrm{H}$-benzo[d]imidazol-1-yl)(naphthalen-2-yl) methanone
M. formula and CHN analyses

M. wt. Rf value \% Yield M.Pt. $\left({ }^{\circ} \mathrm{C}\right)$

$\mathrm{C}_{25} \mathrm{H}_{16} \mathrm{~N}_{4} \mathrm{O}_{4}$ : Anal calcd: $\mathrm{C}, 68.80 ; \mathrm{H}, 3.70 ; \mathrm{N}, 12.84$;

$436.420 .64^{b}$

65

134-136

Found: C, 68.83; H, 3.72; N, 12.87

$\mathrm{C}_{21} \mathrm{H}_{13} \mathrm{~N}_{5} \mathrm{O}_{5}$ : Anal calcd: $\mathrm{C}, 60.72 ; \mathrm{H}, 3.15 ; \mathrm{N}, 16.86$; Found: $\mathrm{C}, 60.75 ; \mathrm{H}, 3.17 ; \mathrm{N}, 16.89$

$415.360 .66^{\mathrm{b}} \quad 74$

$119-121$ 
Table 2 Anticancer screening results of synthesized compounds

\begin{tabular}{lclr}
\hline Comp. & MCF-7 cell line & Comp. & MCF-7 cell line \\
\hline Anticancer screening $\left(/ C_{50}=\mu M\right)$ & & \\
$\mathbf{1}$ & 31.0 & $\mathbf{1 6}$ & 231.8 \\
$\mathbf{2}$ & 41.8 & $\mathbf{1 7}$ & 39.0 \\
$\mathbf{3}$ & 170.6 & $\mathbf{1 8}$ & 161.1 \\
$\mathbf{4}$ & 101.1 & $\mathbf{1 9}$ & 15.8 \\
$\mathbf{5}$ & 67.6 & $\mathbf{2 0}$ & 23.6 \\
$\mathbf{6}$ & 378.3 & $\mathbf{2 1}$ & 5.4 \\
$\mathbf{7}$ & 112.7 & $\mathbf{2 2}$ & 00.9 \\
$\mathbf{8}$ & $>628.1$ & $\mathbf{2 3}$ & 50.8 \\
$\mathbf{9}$ & 310.4 & $\mathbf{2 4}$ & 61.9 \\
$\mathbf{1 0}$ & 157.9 & $\mathbf{2 5}$ & 18.8 \\
$\mathbf{1 1}$ & 321.2 & $\mathbf{2 6}$ & 176.0 \\
$\mathbf{1 2}$ & 7.0 & $\mathbf{2 7}$ & 123.1 \\
$\mathbf{1 3}$ & 19.1 & $\mathbf{2 8}$ & 31.7 \\
$\mathbf{1 4}$ & 394.9 & $\mathbf{2 9}$ & 5.5 \\
$\mathbf{1 5}$ & 11.7 & $\mathbf{3 0}$ & 138.6 \\
5-Fluorouracil & 35.4 & $5-F l u o r o u r a c i l$ & 35.4 \\
\hline
\end{tabular}

${ }^{13} \mathrm{C}$ NMR spectra of the synthesized compounds were recorded on Bruker Advance-II 400 NMR spectrometer with DMSO as a solvent and the chemical shift data were expressed as delta values related to tetramethylsilane. Mass spectra were recorded using Waters, Q-TOF micromass spectrometer.

\section{Procedure for the title compounds (1-11)}

2-Aminobenzimidazole $(0.01 \mathrm{~mol})$ was refluxed with different substituted aromatic aldehyde $(0.01 \mathrm{~mol})$ in ethanol $(20 \mathrm{ml})$ for $4-5 \mathrm{~h}(\mathrm{RT})$ in presence of glacial acetic acid (few drops). Then the reaction mixture was allowed to cool at RT and the precipitated compound was filtered and dried [12].

\section{Synthesis of 2-(alkyl/arylideneamino)-1H-benzo[d] imidazol-1-yl-alkyl/aryl-methanones (12-30)}

Compound of Schiff's bases (1-11) (0.005 mol) were stirred at RT with different acylchlorides $(0.005 \mathrm{~mol})$ in dimethylformamide for $24 \mathrm{~h}$ with the addition of small amount of triethylamine. The resulting reaction mixture was precipitated using ice cold water and the crude product was filtered through a vacuum pump, washed with cold water, dried and recrystallized using rectified spirit [13].

\section{Spectral data of synthesized compounds}

(E) - N-((E) - 3-(4-(Dimethylamino)phe nyl) allylidene)-1H-benzo[d]imidazol-2-amine (1) IR ( $\mathrm{KBr}$ $\mathrm{cm}^{-1}$ ): 1550 ( $\mathrm{N}=\mathrm{CH}$ str.), 3475 (N-H str.), 1431 (Ar., $\mathrm{C}=\mathrm{C}$ str. $), 1253$ (C-N str. $), 1300\left(-\mathrm{N}\left(\mathrm{CH}_{3}\right)_{2}\right.$ str $) ;{ }^{1} \mathrm{H}$ NMR
(DMSO): 9.557-9.575 (d, 1H, N=CH), 6.646-6.714 (d, $1 \mathrm{H},-\mathrm{CH}=\mathrm{CH}), 6.416-7.614(\mathrm{~m}, 8 \mathrm{H}, \mathrm{ArH}), 3.426(\mathrm{~s}, 6 \mathrm{H}$, $\left.\left(\mathrm{CH}_{3}\right)_{2}\right) ;{ }^{13} \mathrm{C}$ NMR (DMSO): 40, 115, 119, 123, 127, 135, 138, 148, 159, 162; MS: $m / z=291.12\left(\mathrm{M}^{+}+1\right)$.

(E)-1-(((1H-Benzo[d]imidazol-2-yl)imino)methyl)naphthalen-2-ol (2) IR $\left(\mathrm{KBr} \mathrm{cm}^{-1}\right): 3066(\mathrm{~N}-\mathrm{H}$ str., of imidazole), 3012 (C-H aromatic ring str.), 1442 (Ar., $\mathrm{C}=\mathrm{C}$ str.), 1550 ( $\mathrm{N}=\mathrm{CH}$ str.), 1253 (C-N str.), 3518 (O-H str.); ${ }^{1} \mathrm{H}$ NMR (DMSO): 10.295 (s, 1H, N=CH), 7.096-8.108 (m, $10 \mathrm{H}, \mathrm{ArH}), 4.481(\mathrm{~s}, 1 \mathrm{H}, \mathrm{OH}), 10.809(\mathrm{~s}, 1 \mathrm{H}, \mathrm{NH}$ of imidazole); ${ }^{13} \mathrm{C}$ NMR (DMSO): 115, 118, 123, 127, 128, 129, $132,135,138,159,162$; MS: $m / z=288.39\left(\mathrm{M}^{+}+1\right)$.

(E)-N-(3,4-Dimethoxybenzylidene)-1H-benzo[d]imidazol-2-amine (3) IR $\left(\mathrm{KBr} \mathrm{cm}{ }^{-1}\right): 3410(\mathrm{~N}-\mathrm{H}$ str.), 3058 (Ar., $\mathrm{C}-\mathrm{H}$ str.), 1542 ( $\mathrm{C}=\mathrm{C}$ str.), 1610 ( $\mathrm{N}=\mathrm{CH}$ str.), 2827 (Ar., $\mathrm{OCH}_{3}$ str.); ${ }^{1} \mathrm{H}$ NMR (DMSO): 9.487 (s, $1 \mathrm{H}, \mathrm{N}=\mathrm{CH}$ ), 6.982-7.849 (m, 7H, ArH), 10.452 (s, 1H, NH of imidazole) $3.502\left(\mathrm{~s}, 6 \mathrm{H},\left(\mathrm{OCH}_{3}\right)_{2}\right) ;{ }^{13} \mathrm{C}$ NMR (DMSO): 56, 115, $123,127,138,152,159,162$; MS: $m / z=282.14\left(\mathrm{M}^{+}+1\right)$.

(E)-4-(((1H-Benzo[d]imidazol-2-yl)imino)methyl)phenol (4) IR $\left(\mathrm{KBr} \mathrm{cm}^{-1}\right): 3440$ (N-H str.), 3063 (Ar., C-H str.), 1537 (C=C str.), 1613 ( $\mathrm{N}=\mathrm{CH}$ str.), 3452 (O-H str.); ${ }^{1} \mathrm{H}$ NMR (DMSO): $9.582(\mathrm{~s}, 1 \mathrm{H}, \mathrm{N}=\mathrm{CH}), 7.106-8.367(\mathrm{~m}$, $8 \mathrm{H}, \mathrm{ArH}), 10.809$ (s, 1H, NH of imidazole); ${ }^{13} \mathrm{C}$ NMR (DMSO): 115, 117, 123, 126, 129, 131, 138, 159, 162; MS: $m / z=238.17\left(\mathrm{M}^{+}+1\right)$.

(E)-N-(4-Nitrobenzylidene)-1H-benzo[d]imidazol-2-amine (5) IR $\left(\mathrm{KBr} \mathrm{cm}^{-1}\right): 3240(\mathrm{~N}-\mathrm{H}$ str., of imidazole ring), 2974 ( $\mathrm{C}-\mathrm{H}$ aromatic ring str.), 1465 (Ar., $\mathrm{C}=\mathrm{C}$ str.), 1550 ( $\mathrm{N}=\mathrm{CH}$ str.), $1548\left(\mathrm{Ar}-\mathrm{C}-\mathrm{NO}_{2}\right.$, asym str.); ${ }^{1} \mathrm{H}$ NMR (DMSO): 9.550 (s, $1 \mathrm{H}, \mathrm{N}=\mathrm{CH}$ ), 7.103$8.105(\mathrm{~m}, 4 \mathrm{H}, \mathrm{ArH}), 8.116-8.376$ (d, 4H, Ar- $\mathrm{NO}_{2}$ ), 12.73 (s, $1 \mathrm{H}, \mathrm{NH}$ of imidazole); ${ }^{13} \mathrm{C}$ NMR (DMSO): 115,120 , $123,130,138,149,159,162 ; \mathrm{MS}: m / z=267.26\left(\mathrm{M}^{+}+1\right)$.

(E)-N-(4-(Dimethylamino)benzylidene)-1H-benzo[d] imidazol-2-amine (6) IR $\left(\mathrm{KBr} \mathrm{cm}{ }^{-1}\right): 1550(\mathrm{~N}=\mathrm{CH}$ str.), 3374 (N-H str.), 1462 (Ar., $\mathrm{C}=\mathrm{C}$ str.), $1298(\mathrm{C}-\mathrm{N}$ str. $\left.-\mathrm{N}\left(\mathrm{CH}_{3}\right)_{2}\right) ;{ }^{1} \mathrm{H}$ NMR (DMSO): $9.206(\mathrm{~s}, 1 \mathrm{H}, \mathrm{N}=\mathrm{CH})$, 6.697-7.823 (m, 8H, ArH), $3.043\left(\mathrm{~s}, 6 \mathrm{H},\left(\mathrm{CH}_{3}\right)_{2}\right), 12.42(\mathrm{~s}$, $1 \mathrm{H}, \mathrm{NH}$ of imidazole); ${ }^{13} \mathrm{C}$ NMR (DMSO): $40,115,123$, 138, 159, 162; MS: $m / z=265.35\left(\mathrm{M}^{+}+1\right)$.

(E)-N-(3-Nitrobenzylidene)-1H-benzo[d]imidazol-2-amine (7) IR $\left(\mathrm{KBr} \mathrm{cm}^{-1}\right): 3428$ (N-H str.), 3068 (Ar., $\mathrm{C}-\mathrm{H}$ str.), 1531 ( $\mathrm{C}=\mathrm{C}$ str.), 1618 ( $\mathrm{C}=\mathrm{N}$ str.), 1547 (Ar-NO $\mathrm{NO}_{2}$ str.); ${ }^{1} \mathrm{H}$ NMR (DMSO): 9.515 (s, 1H, $\mathrm{N}=\mathrm{CH}), 7.213-8.378(\mathrm{~m}, 8 \mathrm{H}, \mathrm{ArH}), 10.23(\mathrm{~s}, 1 \mathrm{H}, \mathrm{NH}$ of 
Table 3 Antimicrobial activity of synthesized compounds

\begin{tabular}{|c|c|c|c|c|c|}
\hline \multirow[t]{3}{*}{ Comp. } & \multicolumn{5}{|c|}{ Antimicrobial screening $(\mathrm{MIC}=\mu \mathrm{M})$} \\
\hline & \multicolumn{3}{|c|}{ Bacterial species } & \multicolumn{2}{|c|}{ Fungal species } \\
\hline & E. coli & B. subtilis & S. aureus & C. albicans & A. niger \\
\hline 1 & 05.4 & 10.7 & 43 & 10.7 & 21.5 \\
\hline 2 & 43.5 & 21.8 & 43.5 & 5.4 & 10.9 \\
\hline 3 & 22.2 & 22.2 & 44.4 & 11.1 & 22.2 \\
\hline 4 & 06.6 & 13.1 & 52.7 & 13.1 & 26.3 \\
\hline 5 & 46.9 & 23.5 & 46.9 & 23.5 & 23.5 \\
\hline 6 & 47.3 & 23.6 & 47.3 & 47.3 & 47.3 \\
\hline 7 & 23.5 & 23.5 & 46.9 & 93.9 & 23.5 \\
\hline 8 & 39.3 & 39.3 & 78.5 & 78.5 & 78.5 \\
\hline 9 & 24.9 & 24.9 & 49.7 & 24.9 & 49.7 \\
\hline 10 & 14.5 & 29 & 58.1 & 29 & 58.1 \\
\hline 11 & 10.0 & 20.1 & 40.1 & 20.1 & 20.1 \\
\hline 12 & 248.5 & 248.5 & 15.5 & 62.1 & 31.1 \\
\hline 13 & 56.1 & 28.1 & 14 & 14 & 7 \\
\hline 14 & 197.4 & 197.4 & 24.7 & 98.7 & 24.7 \\
\hline 15 & 194.3 & 194.3 & 24.3 & 48.6 & 24.3 \\
\hline 16 & 58.0 & 29 & 14.5 & 14.5 & 7.2 \\
\hline 17 & 28.7 & 28.7 & 14.4 & 28.7 & 7.2 \\
\hline 18 & 26.9 & 13.4 & 13.4 & 26.9 & 6.7 \\
\hline 19 & 49.5 & 12.4 & 12.4 & 6.2 & 3.1 \\
\hline 20 & 45.5 & 11.4 & 22.7 & 22.7 & 11.4 \\
\hline 21 & 27.9 & 14 & 14 & 55.9 & 7 \\
\hline 22 & 56.9 & 28.4 & 14.2 & 28.4 & 7.1 \\
\hline 23 & 60.5 & 30.2 & 15.1 & 30.2 & 7.5 \\
\hline 24 & 28.6 & 28.6 & 14.3 & 28.6 & 7.1 \\
\hline 25 & 60.2 & 30.1 & 15 & 30.1 & 7.5 \\
\hline 26 & 29.7 & 29.7 & 14.9 & 59.5 & 7.4 \\
\hline 27 & 58.1 & 29 & 14.5 & 29 & 7.2 \\
\hline 28 & 49.5 & 24.8 & 24.8 & 24.8 & 12.4 \\
\hline 29 & 47.3 & 11.8 & 23.6 & 23.6 & 11.8 \\
\hline 30 & 29.9 & 29.9 & 14.9 & 59.7 & 7.5 \\
\hline DMSO & NA & NA & NA & NA & NA \\
\hline Std. drugs & $4.7^{a}$ & $4.7^{\mathrm{a}}$ & $4.7^{\mathrm{a}}$ & $5.1^{b}$ & $5.1^{b}$ \\
\hline
\end{tabular}

NA no activity, DMSO dimethyl sulphoxide

Std. drugs: ${ }^{\mathrm{a}}$ Norfloxacin, ${ }^{\mathrm{b}}$ Fluconazole

imidazole); ${ }^{13} \mathrm{C}$ NMR (DMSO): 115,123, 127, 135, 138, 150, 159, 162; MS: $m / z=267.28\left(\mathrm{M}^{+}+1\right)$.

(E)-N-Ethylidene-1H-benzo[d]imidazol-2-amine (8) IR $\left(\mathrm{KBr} \mathrm{cm}^{-1}\right)$ : 3267 (N-H str., of imidazole ring), $2924(\mathrm{C}-\mathrm{H}$ aromatic ring str.), 1465 (Ar., $\mathrm{C}=\mathrm{C}$ str.), $1550(\mathrm{~N}=\mathrm{CH}$ str.), 2877 ( $\mathrm{R}^{-\mathrm{CH}_{3}}$, sym str.); ${ }^{1} \mathrm{H}$ NMR (DMSO): 8.654 (s, $1 \mathrm{H}$, $\mathrm{N}=\mathrm{CH}), 6.897-7.143(\mathrm{~m}, 4 \mathrm{H}, \mathrm{ArH}), 1.243\left(\mathrm{~s}, 3 \mathrm{H}, \mathrm{CH}_{3}\right) ;{ }^{13} \mathrm{C}$ NMR: 22, 111, 120, 154, 175. MS: $m / z=160.28\left(\mathrm{M}^{+}+1\right)$.

(E)-N-(4-Methoxybenzylidene)-1H-benzo[d]imidazol-2-amine (9) IR $\left(\mathrm{KBr} \mathrm{cm}^{-1}\right): 3340(\mathrm{~N}-\mathrm{H}$ str., of imidazole ring), 2970 ( $\mathrm{C}-\mathrm{H}$ aromatic ring str.), 1496 (Ar., $\mathrm{C}=\mathrm{C}$ str.), 1566 ( $\mathrm{N}=\mathrm{CH}$ str.), $1257\left(\mathrm{C}-\mathrm{O}-\mathrm{C}\right.$ str.); ${ }^{1} \mathrm{H}$ NMR (DMSO): 9.383 (s, 1H, N=CH), 7.027-7.960 (m, $8 \mathrm{H}, \mathrm{ArH}), 3.523\left(\mathrm{~s}, 3 \mathrm{H}, \mathrm{OCH}_{3}\right), 12.497(\mathrm{~s}, 1 \mathrm{H}, \mathrm{NH}$ of imidazole); ${ }^{13} \mathrm{C}$ NMR (DMSO): 57, 114, 115, 123, 126, 130, 138, 162; MS: $m / z=252.28\left(\mathrm{M}^{+}+1\right)$.

(E)-5-((1H-Benzo[d]imidazol-2-yl)imino)pentanal (10) IR ( $\left.\mathrm{KBr} \mathrm{cm}^{-1}\right): 3426$ (N-H str.), 3054 (Ar., C-H str.), 1562 ( $\mathrm{C}=\mathrm{C}$ str.), 1623 ( $\mathrm{N}=\mathrm{CH}$ str.), 2773 (Aliphatic $\mathrm{C}-\mathrm{H}$ str.), 1724 (Aliphatic aldehyde $\mathrm{C}=\mathrm{O}$ str.); ${ }^{1} \mathrm{H}$ NMR (DMSO): $8.454(\mathrm{t}, 1 \mathrm{H}, \mathrm{N}=\mathrm{CH}), 6.856-7.143(\mathrm{~m}, 4 \mathrm{H}$, $\mathrm{ArH}), 1.243-2.567\left(\mathrm{~m}, 6 \mathrm{H}, \mathrm{CH}_{2}\right), 9.700(\mathrm{t}, 1 \mathrm{H}, \mathrm{CH}=\mathrm{O})$; ${ }^{13} \mathrm{C}$ NMR (DMSO): $18,28,44,115,123,138,160,162$, 202. MS: $m / z=216.26\left(\mathrm{M}^{+}+1\right)$.

(E)-N-(3,4,5-Trimethoxybenzylidene)-1H-benzo[d]imidazol-2-amine (11) IR: 3429 (N-H str.), 3064 (Ar., C-H str.), 1577 ( $\mathrm{C}=\mathrm{C}$ str.), 1606 ( $\mathrm{N}=\mathrm{CH}$ str.), 2835 (Ar., O$\mathrm{CH}_{3}$ str.); ${ }^{1} \mathrm{H}$ NMR (DMSO): 9.476 (s, $\left.1 \mathrm{H}, \mathrm{N}=\mathrm{CH}\right), 6.962-$ 7.859 (m, 6H, ArH), 10.462 (s, 1H, NH of imidazole) 3.382 (s, 9H, $\left.\left(\mathrm{OCH}_{3}\right)_{3}\right) ;{ }^{13} \mathrm{C}$ NMR (DMSO): 56, 106, 115, 123, 127, 138, 141, 152, 159, 162; MS: $m / z=312.14\left(\mathrm{M}^{+}+1\right)$.

(E)-1-(2-(Ethylideneamino)-1H-benzo[d]imidazol-1-yl) ethanone (12) IR ( $\left.\mathrm{KBr} \mathrm{cm}{ }^{-1}\right): 1661(\mathrm{C}=\mathrm{O}$ str.), 2919 (C-H aromatic str.), $1575(\mathrm{~N}=\mathrm{CH}$ str.), $2849(\mathrm{CH}$ str. (sym), $\mathrm{R}_{-} \mathrm{CH}_{3}$ ); ${ }^{1} \mathrm{H}$ NMR (DMSO): 7.305-7.627 (m, 4H, $\mathrm{Ar}-\mathrm{H}), 7.233(\mathrm{~s}, 1 \mathrm{H}, \mathrm{N}=\mathrm{CH}), 1.273-1.276\left(\mathrm{~d}, 3 \mathrm{H}, \mathrm{CH}_{3}\right)$, 2.856 (s, $\left.3 \mathrm{H}, \mathrm{CH}_{3}\right) ;{ }^{13} \mathrm{C}$ NMR (DMSO): $16,24,115,123$, 129, 138, 141, 162, 168; MS: $m / z=201\left(\mathrm{M}^{+}+1\right)$.

(E)-(3,5-Dinitrophenyl)(2-((4-methoxy-benzylidene) amino)-1H-benzo[d]imidazol-1-yl)methanone (13) IR ( $\left.\mathrm{KBr} \mathrm{cm}^{-1}\right)$ : 1710 (C=O str.), 2924 (C-H aromatic str.), 1537 ( $\mathrm{N}=\mathrm{CH}$ str.), 1545 (Ar- $\mathrm{NO}_{2}$ str.), 1110 (C-O-C str., $\left.\mathrm{OCH}_{3}\right) ;{ }^{1} \mathrm{H}$ NMR (DMSO): 6.785-7.943 (m, 8H, ArH), $8.632(\mathrm{~s}, 1 \mathrm{H}, \mathrm{N}=\mathrm{CH}), 2.984\left(\mathrm{~s}, 3 \mathrm{H},\left(\mathrm{OCH}_{3}\right), 8.912-9.063\right.$ (m, 3H, $\left.\operatorname{Ar}\left(\mathrm{NO}_{2}\right)_{2}\right) ;{ }^{13} \mathrm{C}$ NMR (DMSO): 56, 115, 123, 125, 130, 150, 163, 168; MS: $m / z=445\left(\mathrm{M}^{+}+1\right)$.

(E)-5-((1-(3,5-Dinitrobenzoyl)-1H-benzo[d]imidazol-2-yl) imino)pentanal (14) $\mathrm{IR} \quad\left(\mathrm{KBr} \quad \mathrm{cm}^{-1}\right): 1701 \quad(\mathrm{C}=\mathrm{O}$ str.), 3122 (C-H aromatic str.), $1627(\mathrm{~N}=\mathrm{CH}$ str.), 1543 (Ar., $\mathrm{NO}_{2}$ str.), 1727 (Aliphatic aldehyde $\mathrm{C}=\mathrm{O}$ str.); ${ }^{1} \mathrm{H}$ NMR (DMSO): 6.875-7.946 (m, 4H, ArH), $8.632(\mathrm{~s}, 1 \mathrm{H}$, $\mathrm{N}=\mathrm{CH}), \quad 8.912-9.063\left(\mathrm{~m}, 3 \mathrm{H}, \operatorname{Ar}\left(\mathrm{NO}_{2}\right)_{2}\right), 9.254-9.678$ (m, 1H, CHO); ${ }^{13} \mathrm{C}$ NMR (DMSO): 19, 28, 44, 115, 123, 125, 130, 150, 163, 168; MS: $m / z=409\left(\mathrm{M}^{+}+1\right)$.

(E)-5-((1-Acetyl-1H-benzo[d]imidazol-2-yl)imino)pentanal (15) IR $\left(\mathrm{KBr} \mathrm{cm}^{-1}\right): 1695$ (C=O str.), $3050(\mathrm{C}-\mathrm{H}$ aromatic str.), $1606\left(\mathrm{~N}=\mathrm{CH}\right.$ str.), $1535\left(\mathrm{C}-\mathrm{NO}_{2}\right.$ str.), 2860 


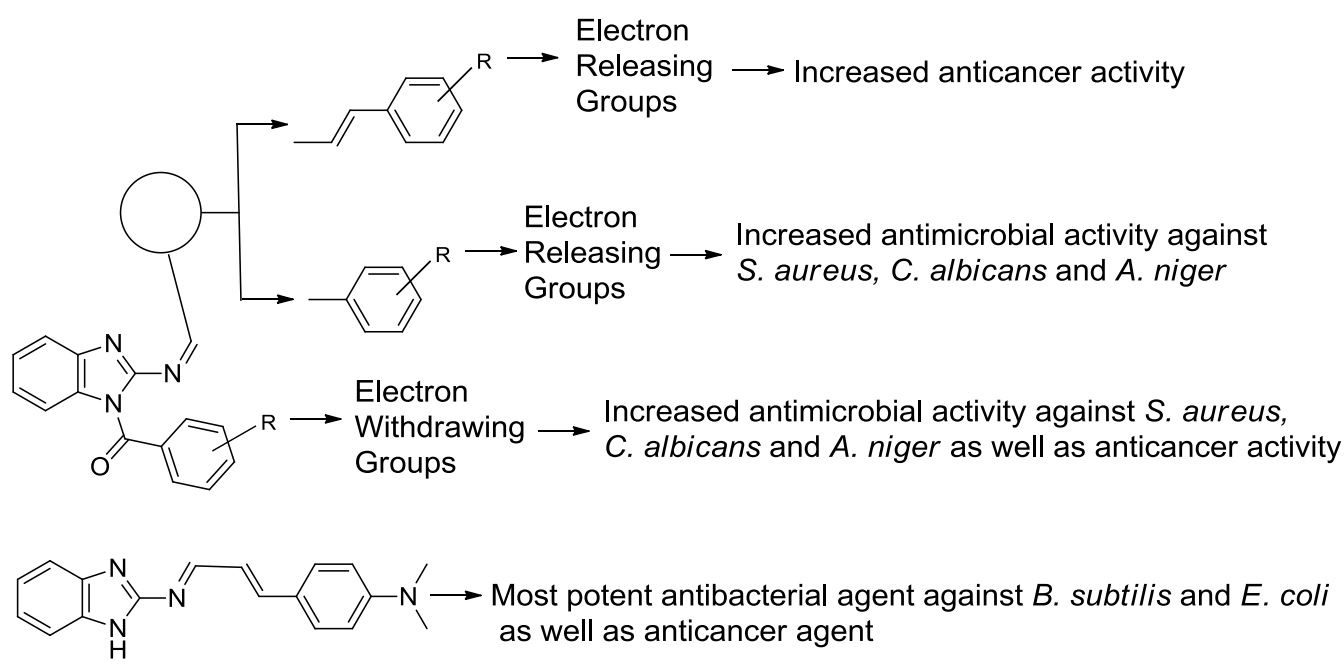

Fig. 1 Structural requirements for antimicrobial and anticancer activity of synthesized benzimidazole derivatives

(C-H sym. str., $\mathrm{R}-\mathrm{CH}_{3}$ ), 1728 (Aliphatic aldehyde $\mathrm{C}=\mathrm{O}$ str.); ${ }^{1} \mathrm{H}$ NMR (DMSO): 7.875-8.246 (m, 4H, ArH), 7.632 $(\mathrm{s}, 1 \mathrm{H}, \mathrm{N}=\mathrm{CH}), 9.254-9.678(\mathrm{~m}, 1 \mathrm{H}, \mathrm{CHO}), 2.856(\mathrm{~s}, 3 \mathrm{H}$, $\left.\mathrm{CH}_{3}\right) ;{ }^{13} \mathrm{C}$ NMR (DMSO): 19, 24, 28, 44, 115, 123, 138, 142, 163, 168, 202; MS: $m / z=257\left(\mathrm{M}^{+}+1\right)$.

(E)-(3,5-Dinitrophenyl)(2-((4-hydroxybenzylidene) amino)-1H-benzo[d]imidazol-1-yl)methanone (16) IR $\left(\mathrm{KBr} \mathrm{cm}{ }^{-1}\right)$ : 1685 (C=O str.), 3094 (C-H aromatic str.), 1630 ( $\mathrm{N}=\mathrm{CH}$ str.), 1544 ( $\mathrm{C}-\mathrm{NO}_{2}$ str.), 3465 (O-H str); ${ }^{1} \mathrm{H}$ NMR (DMSO): 6.885-7.632 (m, 8H, ArH), 8.654 (s, $1 \mathrm{H}, \mathrm{N}=\mathrm{CH}), 8.912-9.063\left(\mathrm{~m}, 3 \mathrm{H}, \mathrm{Ar}\left(\mathrm{NO}_{2}\right)_{2}\right) ;{ }^{13} \mathrm{C} \mathrm{NMR}$ (DMSO): 115, 123, 125, 130, 132, 138, 150, 160, 168; MS: $m / z=431\left(\mathrm{M}^{+}+1\right)$.

(E)-(2-((2,4-Dimethoxybenzylidene)amino)-1H-benzo[d] imidazol-1-yl)(naphthalen-2-yl)methanone (17) IR ( $\left.\mathrm{KBr} \mathrm{cm}^{-1}\right)$ : 1695 (C=O str.), 2919 (C-H aromatic str.), 1634 ( $\mathrm{N}=\mathrm{CH}$ str.), 2850 (Ar., $\mathrm{OCH}_{3}$ str.); 1646 (naph. ring str.); ${ }^{1} \mathrm{H}$ NMR (DMSO): 6.844-8.213 (m, 14H, ArH), $8.612(\mathrm{~s}, 1 \mathrm{H}, \mathrm{N}=\mathrm{CH}), 2.804\left(\mathrm{~s}, 6 \mathrm{H},\left(\mathrm{OCH}_{3}\right)_{2}\right) ;{ }^{13} \mathrm{C} \mathrm{NMR}$ (DMSO): 56, 101, 107, 109, 115, 123, 127, 129, 132, 138, 142, 160, 168; MS: $m / z=435\left(\mathrm{M}^{+}+1\right)$.

(E)-Naphthalen-2-yl(2-((3,4,5-trimethoxybenzylidene) amino)-1H-benzo[d]imidazol-1-yl)methanone (18) IR ( $\left.\mathrm{KBr} \mathrm{cm}^{-1}\right)$ : 1691 ( $\mathrm{C}=\mathrm{O}$ str.), 1548 ( $\mathrm{N}=\mathrm{CH}$ str.), 1140 $\left(\mathrm{C}-\mathrm{O}-\mathrm{C}\right.$ str., $\left.\mathrm{OCH}_{3}\right) ; 795(\mathrm{C}-\mathrm{H}$ out of plane bending, naphthalene ring); ${ }^{1} \mathrm{H}$ NMR (DMSO): 8.590 (s, $1 \mathrm{H}$, $\mathrm{N}=\mathrm{CH}), 4.194\left(\mathrm{~s}, 9 \mathrm{H},\left(\mathrm{OCH}_{3}\right)_{3}\right), 6.971-8.070(\mathrm{~m}, 11 \mathrm{H}$, Ar-H); ${ }^{13} \mathrm{C}$ NMR (DMSO): 57, 107, 115, 123, 124, 127, $128,131,139,142,151,160,168$; MS: $m / z=465\left(\mathrm{M}^{+}+1\right)$.
(E)-(3,5-Dinitrophenyl)(2-((3,4,5-trimethoxybenzylidene) amino)-1H-benzo[d]imidazol-1-yl)methanone (19) IR ( $\left.\mathrm{KBr} \mathrm{cm}^{-1}\right)$ : $1681(\mathrm{C}=\mathrm{O}$ str.), 1539 ( $\mathrm{N}=\mathrm{CH}$ str.), 2850 $\left(\mathrm{CH}_{3}\right.$ sym. str., R-OCH$\left.{ }_{3}\right) ; 1345\left(\mathrm{C}-\mathrm{NO}_{2}\right.$ str.); ${ }^{1} \mathrm{H}$ NMR (DMSO): $8.947(\mathrm{~s}, 1 \mathrm{H}, \mathrm{N}=\mathrm{CH}), 3.955\left(\mathrm{~s}, 9 \mathrm{H},\left(\mathrm{OCH}_{3}\right)_{3}\right)$, 9.860-9.865 (m, 3H, Ar- $\left.(\mathrm{NO})_{2}\right), 7.948-7.951(\mathrm{~d}, 2 \mathrm{H}$, Ar-H), 7.343-7.366 (m, 2H, Ar-H); ${ }^{13} \mathrm{C}$ NMR (DMSO): 57, 106, 115, 125, 128, 129, 131, 139, 142, 147, 151, 168; MS: $m / z=505\left(\mathrm{M}^{+}+1\right)$.

(E) - 1 - (2-((3,4,5-trime thoxybenzylidene) amino)-1H-benzo[d]imidazol-1-yl)hexadecan-1-one (20) IR ( $\left.\mathrm{KBr} \mathrm{cm}^{-1}\right)$ : 1685 ( $\mathrm{C}=\mathrm{O}$ str.), 3061 ( $\mathrm{C}-\mathrm{H}$ aromatic str.), 1623 ( $\mathrm{N}=\mathrm{CH}$ str.), 2843 (Ar., $\mathrm{O}-\mathrm{CH}_{3}$ str.), 1266 (Palmitoyl group str.); ${ }^{1} \mathrm{H}$ NMR (DMSO): 7.283$7.286(\mathrm{~m}, 6 \mathrm{H}, \mathrm{ArH}), 1.278-2.386\left(\mathrm{~m}, 28 \mathrm{H}, \mathrm{CH}_{2}\right.$ of palmitoyl), 0.884-0.903 (t, 3H, $\left.\mathrm{CH}_{3}\right), 3.264\left(\mathrm{~s}, 9 \mathrm{H},\left(\mathrm{OCH}_{3}\right)_{3}\right)$; ${ }^{13} \mathrm{C}$ NMR (DMSO): 14, 23, 26, 30, 32, 56, 106, 115, 123, 128, 130, 139, 142, 160, 170; MS: $m / z=549\left(\mathrm{M}^{+}+1\right)$.

(E)-(3-Nitrophenyl)(2-((4-(pyridin-2-yl)benzylidene) amino)-1H-benzo[d]imidazol-1-yl) methanone (21) IR $\left(\mathrm{KBr} \mathrm{cm}{ }^{-1}\right)$ : 1682 (C=O str.), 2922 (C-H aromatic str.), 1525 ( $\mathrm{N}=\mathrm{CH}$ str.), 1557 ( $\mathrm{C}=\mathrm{C}$ and $\mathrm{C}=\mathrm{N}$ str. of pyridine ring), 1543 (C- $\mathrm{NO}_{2}$ str.); ${ }^{1} \mathrm{H}$ NMR (DMSO): 10.019 (s, $1 \mathrm{H}, \mathrm{N}=\mathrm{CH}$ ), 7.305-8.658 (m, 15H, ArH), $8.662(\mathrm{~s}, 1 \mathrm{H}$, $\left.\mathrm{Ar}-\mathrm{NO}_{2}\right) ;{ }^{13} \mathrm{C}$ NMR (DMSO): 115, 123, 126, 129, 130, 132, 142, 150, 155, 160, 168; MS: $m / z=447\left(\mathrm{M}^{+}+1\right)$.

(2-((E)-((E)-3-(4-(Dimethylamino)phenyl)allylidene) amino)-1H-benzo[d]imidazol-1-yl)(3-nitrophenyl)methanone (22) IR $\left(\mathrm{KBr} \mathrm{cm}{ }^{-1}\right): 1723(\mathrm{C}=\mathrm{O}$ str.), $2920(\mathrm{C}-\mathrm{H}$ aromatic str.), 1530 ( $\mathrm{N}=\mathrm{CH}$ str.), 1549 (Ar- $\mathrm{NO}_{2}$ str.), 1349 (C-N str., of ter. arylamine); ${ }^{1} \mathrm{H}$ NMR (DMSO): 
8.390-8.410 (d, $1 \mathrm{H}, \quad \mathrm{N}=\mathrm{CH}), \quad 6.731-6.740(\mathrm{~d}, 1 \mathrm{H},-$ $\mathrm{CH}=\mathrm{CH}), 6.250-8.355(\mathrm{~m}, 11 \mathrm{H}, \operatorname{ArH}), 8.919(\mathrm{~s}, 1 \mathrm{H}$, Ar- $\left.\mathrm{NO}_{2}\right) 3.559$ (s, 6H, $\left.\left(\mathrm{CH}_{3}\right)_{2}\right) ;{ }^{13} \mathrm{C}$ NMR (DMSO): 40, 115, 123, 127, 131, 138, 149, 164, 168; MS: $m / z=439\left(\mathrm{M}^{+}+1\right)$.

(E)-(2-((4-(Dimethylamino)benzylidene) amino)-1H-benzo[d]imidazol-1-yl)(3-nitrophenyl) methanone (23) IR $\left(\mathrm{KBr} \mathrm{cm}^{-1}\right)$ : $1719(\mathrm{C}=\mathrm{O}$ str.), $3085(\mathrm{C}-\mathrm{H}$ aromatic str.), 1615 ( $\mathrm{N}=\mathrm{CH}$ str.), $1545\left(\mathrm{Ar}-\mathrm{NO}_{2}\right.$ str.), 1514 (C-N str.); ${ }^{1} \mathrm{H}$ NMR (DMSO): 7.169-8.987 (m, 12H, ArH), $9.568(\mathrm{~s}, 1 \mathrm{H}, \mathrm{N}=\mathrm{CH}), 2.909\left(\mathrm{~s}, 6 \mathrm{H}\left(\mathrm{CH}_{3}\right)_{2}\right) ;{ }^{13} \mathrm{C} \mathrm{NMR}$ (DMSO): 40, 115, 123, 127, 131, 138, 149, 160, 168; MS: $m / z=413\left(\mathrm{M}^{+}+1\right)$.

(E)-(2-(((2-hydroxynaphthalen-1-yl)-methylene) amino)-1H-benzo[d]imidazol-1-yl)(3-nitrophenyl)methanone (24) IR $\left(\mathrm{KBr} \mathrm{cm}^{-1}\right): 1696(\mathrm{C}=\mathrm{O}$ str.), $2924(\mathrm{C}-\mathrm{H}$ aromatic str.), 1553 ( $\mathrm{N}=\mathrm{CH}$ str.), $1546\left(\mathrm{Ar}-\mathrm{NO}_{2}\right.$ str.), 752 (O-H bending (out of plane)); ${ }^{1} \mathrm{H}$ NMR (DMSO): 6.7488.632 (m, 14H, ArH), 9.652 (s, $1 \mathrm{H}, \mathrm{N}=\mathrm{CH}) ;{ }^{13} \mathrm{C} \mathrm{NMR}$ (DMSO):115, 118, 123, 125, 127, 128, 131, 138, 149, 160, 168; MS: $m / z=436\left(\mathrm{M}^{+}+1\right)$.

(E)-(2-((4-Nitrobenzylidene)amino)-1H-benzo[d]imidazol-1-yl)(3-nitrophenyl)methanone (25) IR $\left(\mathrm{KBr} \mathrm{cm}^{-1}\right)$ : $1704(\mathrm{C}=\mathrm{O}$ str.), 3107 (C-H aromatic str.), $1617(\mathrm{~N}=\mathrm{CH}$ str.), 1549 (Ar., $\mathrm{NO}_{2}$ str.); ${ }^{1} \mathrm{H}$ NMR (DMSO): 7.206-8.689 (m, 12H, ArH), 9.672 (s, 1H, N=CH); ${ }^{13} \mathrm{C}$ NMR (DMSO): 115, 121, 123, 125, 127, 131, 136, 139, 151, 160, 168; MS: $m / z=415\left(\mathrm{M}^{+}+1\right)$.

(E)-Naphthalen-2-yl(2-((4-nitrobenzylidene) amino)-1H-benzo[d]imidazol-1-yl)methanone (26) IR $\left(\mathrm{KBr} \mathrm{cm}{ }^{-1}\right)$ : 1686 ( $\mathrm{C}=\mathrm{O}$ str.), $3056(\mathrm{C}-\mathrm{H}$ aromatic str.), 1600 ( $\mathrm{N}=\mathrm{CH}$ str.), 1545 (Ar., $\mathrm{NO}_{2}$ str.), 1592 (Naphthalene ring str.); ${ }^{1} \mathrm{H}$ NMR (DMSO): 6.865-7.954 (m, 11H, ArH), $8.765(\mathrm{~s}, 1 \mathrm{H}, \mathrm{N}=\mathrm{CH}), 8.923-8.967\left(\mathrm{~m}, 4 \mathrm{H}, \operatorname{Ar}\left(\mathrm{NO}_{2}\right) ;{ }^{13} \mathrm{C}\right.$ NMR (DMSO): 115, 123, 124, 128, 129, 131, 135, 136, 139, 142, 149, 160, 168; MS: $m / z=420\left(\mathrm{M}^{+}+1\right)$.

(E)-(2-((3,4-Dimethoxybenzylidene)amino)-1H-benzo[d] imidazol-1-yl)(3-nitro phenyl)methanone (27) IR ( $\mathrm{KBr}$ $\mathrm{cm}^{-1}$ ): 1684 (C=O str.), 1611 ( $\mathrm{N}=\mathrm{CH}$ str.), 2875 (Ar., O$\mathrm{CH}_{3}$ str.); 1543 (Ar., $\mathrm{NO}_{2}$ str.); ${ }^{1} \mathrm{H}$ NMR (DMSO): $7.463-$ $8.932(\mathrm{~m}, 11 \mathrm{H}, \mathrm{ArH}), 8.185(\mathrm{~s}, 1 \mathrm{H}, \mathrm{N}=\mathrm{CH}), 2.904(\mathrm{~s}, 6 \mathrm{H}$, $\left.\left(\mathrm{OCH}_{3}\right)_{2}\right) ;{ }^{13} \mathrm{C}$ NMR (DMSO): 56, 115, 123, 125, 127, 136, 139, 142, 147, 150, 152, 160, 168; MS: $m / z=430\left(\mathrm{M}^{+}+1\right)$.

(E)-1-(2-((4-Nitrobenzylidene)amino)-1H-benzo[d]imidazol-1-yl)hexadecan-1-one (28) IR $\left(\mathrm{KBr} \mathrm{cm}^{-1}\right): 1685$ ( $\mathrm{C}=\mathrm{O}$ str.), 2954 ( $\mathrm{C}-\mathrm{H}$ aromatic str.), $1618(\mathrm{~N}=\mathrm{CH}$ str.), 1271 (Palmitoyl group. str.), 1547 (Ar- $\mathrm{NO}_{2}$ str.); ${ }^{1} \mathrm{H}$ NMR (DMSO): 7.624-8.163 (m, 8H, ArH), 8.672 (s, 1H,
$\mathrm{N}=\mathrm{CH}), 1.243-2.496\left(\mathrm{~m}, 28 \mathrm{H}, \mathrm{CH}_{2}\right.$ of palmitoyl), $0.845-$ $0.878\left(\mathrm{t}, 3 \mathrm{H}, \mathrm{CH}_{3}\right) ;{ }^{13} \mathrm{C}$ NMR (DMSO): $14,23,26,30,32$, $56,106,115,120,123,125,128,131,135,136,139,142$, 149, 160, 170; MS: $m / z=504\left(\mathrm{M}^{+}+1\right)$.

(E)-1-(2-((4-(Dimethylamino)benzylidene) amino)-1H-benzo[d]imidazol-1-yl)hexadecan-1-one (29) IR ( $\left.\mathrm{KBr} \mathrm{cm}{ }^{-1}\right): 2927(\mathrm{C}-\mathrm{H}$, aromatic str.), 2813 (C-H str. aliphatic), 1659 ( $\mathrm{C}=\mathrm{O}$ str.), 1594 ( $\mathrm{N}=\mathrm{CH}$ str.), 1303 (C-N str.), 1278 (palmitoyl group str.); ${ }^{1} \mathrm{H}$ NMR (DMSO): 7.878-7.901 (d, $1 \mathrm{H}, \mathrm{N}=\mathrm{CH}), 6.606-6.622(\mathrm{~d}$, $1 \mathrm{H},-\mathrm{CH}=\mathrm{CH}), 6.661-7.519(\mathrm{~m}, 8 \mathrm{H}, \mathrm{ArH}), 3.773(\mathrm{~s}, 6 \mathrm{H}$, $\left.\left(\mathrm{CH}_{3}\right)_{2}\right), 1.252-2.368\left(\mathrm{~m}, 28 \mathrm{H}, \mathrm{CH}_{2}\right.$ of palmitoyl), $0.861-$ $0.894\left(\mathrm{t}, 3 \mathrm{H}, \mathrm{CH}_{3}\right) ;{ }^{13} \mathrm{C}$ NMR (DMSO): $14,23,26,30,32$, $56,106,115,120,123,125,128,130,139,142,149,164,170$; MS: $m / z=528\left(\mathrm{M}^{+}+1\right)$.

(E)-(2-((4-(Dimethylamino)benzylidene) amino)-1H-benzo[d]imidazol-1-yl)(naphthalen-2-yl)methanone (30) $\mathrm{IR}\left(\mathrm{KBr} \mathrm{cm}^{-1}\right)$ : $1683(\mathrm{C}=\mathrm{O}$ str.), $3054(\mathrm{C}-\mathrm{H}$ aromatic str.), 1608 ( $\mathrm{N}=\mathrm{CH}$ str.), 1521 (Ar. $\mathrm{NO}_{2}$ str.), 1448 (C-N str.), 778 ( $\mathrm{C}-\mathrm{H}$ out of plane bending, naphthalene ring); ${ }^{1} \mathrm{H}$ NMR (DMSO): 6.668-7.985 (m, 15H, ArH), 9.584 (s, $1 \mathrm{H}, \mathrm{N}=\mathrm{CH}), 2.909\left(\mathrm{~s}, 6 \mathrm{H}\left(\mathrm{CH}_{3}\right)_{2}\right) ;{ }^{13} \mathrm{C} \mathrm{NMR}$ (DMSO): 40, 115, 123, 124, 128, 130, 139, 142, 160, 168; MS: $m / z=418\left(\mathrm{M}^{+}+1\right)$.

\section{Biological evaluation In vitro antimicrobial assay}

Tube dilution method [15] was used to determine the antimicrobial activity of synthesized compounds against Gram-positive bacteria: Staphylococcus aureus (MTCC3160); Bacillus subtilis (MTCC-441), the Gram-negative bacterium Escherichia coli (MTCC-443) and fungal species: Candida albicans (MTCC-227) and Aspergillus niger (MTCC-281). Dilutions were made for test and standard compounds in appropriate double strength nutrient broth-I.P. (bacteria) or Sabouraud dextrose broth-I.P. (fungi) [16]. The test and standard compounds were incubated at $37^{\circ} \mathrm{C}$ for $24 \mathrm{~h}$ (bacteria), at $25^{\circ} \mathrm{C}$ for 7 days (A. niger) and at $37^{\circ} \mathrm{C}$ for $48 \mathrm{~h}$ (C. albicans) and the minimum inhibitory concentration (MIC) was recorded in $\mu \mathrm{g} / \mathrm{mL}$.

\section{In vitro anticancer assay}

The in vitro anticancer activity of the developed compounds was performed by the Sulforhodamine B (SRB) assay as described by Skehan et al. [14]. The optimal MCF-7 cell count was seeded on flat-bottom well plates and allowed to attach overnight. The compounds (20 $\mu \mathrm{L}$ ) were added in quadruplicates and incubated for $72 \mathrm{~h}$ 
(both drug-free control and treated cells). Cells in each well were fixed with $200 \mu \mathrm{L}$ of $10 \%$ cold trichloroacetic acid. After incubation for $30 \mathrm{~min}$, the individual wells were rinsed with water, allowed to stain in $100 \mu \mathrm{L} 0.4 \%$ SRB [Sigma-Aldrich, St Louis, Missouri, USA] ( $w / v$; in $1 \%$ acetic acid) for $15 \mathrm{~min}$. The air-dried plates were placed on a plate shaker and bound SRB was solubilised in 100 $\mu \mathrm{L} 10 \mathrm{mM}$ Tris base solution. Absorbance was measured using a spectrophotometer at $570 \mathrm{~nm}$ and a doseresponse curve was plotted from which the $\mathrm{IC}_{50}$ value of each compound against each cell type was determined.

\section{Conclusion}

In conclusion, a series of 1,2-disubstituted benzimidazole derivatives were synthesized and assessed for in vitro antimicrobial and anticancer activities against five representative microbial species and cancer cell line. Antimicrobial activity results indicated that the synthesized compound 1 has promising activity towards Gram negative bacteria E. coli. None of the compound showed more potent activity against Gram positive bacteria B. subtilis and S. aureus when compared to reference drug norfloxacin. Moreover, compounds 2 and 19 showed interesting results against fungal strains $C$. albicans and A. niger and comparable to fluconazole. The results from anticancer activity indicated that compounds 12, 21, 22 and 29 showed promising activity against MCF7. These active compounds may be taken as lead compounds for discovery of novel antimicrobial and anticancer agents in future.

\section{Authors' contributions}

SSS, BN, NV and SK have designed, synthesized and carried out the antimicrobial activity and SML, SAAS, KR and VM have carried out the spectral analysis, interpretation and cytotoxicity study of synthesized compounds. All authors read and approved the final manuscript.

\section{Author details \\ ${ }^{1}$ SGT College of Pharmacy, Shree Guru Gobind Singh Tricentenary (SGT) University, Gurugram 122505, India. ${ }^{2}$ University School of Chemical Technol- ogy, Guru Gobind Singh Indraprastha University, Sector-16C, Dwarka, New Delhi 110078 , India. ${ }^{3}$ Faculty of Pharmaceutical Sciences, Maharshi Dayanand University, Rohtak 124001, India. ${ }^{4}$ Faculty of Pharmacy, Universiti Teknologi MARA (UiTM), 42300 Bandar Puncak Alam, Selangor Darul Ehsan, Malaysia. ${ }^{5}$ Collaborative Drug Discovery Research (CDDR) Group, Pharmaceutical Life Sciences Community of Research, Universiti Teknologi MARA (UiTM), 40450 Shah Alam, Selangor Darul Ehsan, Malaysia. ${ }^{6}$ Atta-ur-Rahman Institute for Natural Products Discovery (AuRIns), Universiti Teknologi MARA, Puncak Alam Campus, 42300 Bandar Puncak Alam, Selangor Darul Ehsan, Malaysia. 7 Department of Pharmacology and Toxicology, College of Pharmacy, Qassim University, Buraidah 51452, Kingdom of Saudi Arabia.}

\section{Acknowledgements}

The authors are thankful to the Dean, University School of Chemical Technology, IP University, New Delhi and Head, Department of Pharmaceutical Sciences, M.D. University, Rohtak, for providing necessary facilities to carry out this research work.

\section{Competing interests}

The authors declare that they have no competing interests.
Ethics approval and consent to participate Not applicable.

Funding

Not applicable.

\section{Publisher's Note}

Springer Nature remains neutral with regard to jurisdictional claims in published maps and institutional affiliations.

Received: 1 August 2018 Accepted: 21 November 2018

Published online: 01 December 2018

\section{References}

1. Grossman TH, Bartels DJ, Mullin S, Gross CH, Parsons JD, Liao Y, Grillot AL, Stamos D, Olson ER, Charifson PS, Mani N (2007) Dual targeting of GyrBand ParE by a novel aminobenzimidazole class of antibacterial compounds. Antimicrob Agents Chemother 51:657-666

2. Hassan GS, Kadry HH, Abou-Seri SM, Ali MM, Mahmoud AEE (2011) Synthesis and in vitro cytotoxic activity of novel pyrazolo[3,4-d]pyrim.idines and related pyrazole hydrazones toward breast adenocarcinoma MCF-7 cell line. Bioorg Med Chem 19:6808-6817

3. Ozkay Y, Tunali Y, Karaca H, Isikdag I (2010) Antimicrobial activity and a SAR study of some novel benzimidazole derivatives bearing hydrazone moiety. Eur J Med Chem 45:3293-3298

4. Arora RK, Kaur N, Bansaln Y, Bansal G (2014) Novel coumarin-benzimidazole derivatives as antioxidants and safer anti-inflammatory agents. Acta Pharm Sin B 4:368-375

5. Alasmary FA, Snelling AM, Alafeefy ME, Awaad S, Karodia N (2015) Synthesis and evaluation of selected benzimidazole derivatives as potential antimicrobial agents. Antimicrob Agents Mol 20:15206-15223

6. Oh S, Kim S, Kong S, Yang G, Lee N, Han D, Goo J, Siqueira-Neto JL, FreitasJunior LH, Song R (2014) Synthesis and biological evaluation of 2,3-dihydroimidazo[1,2-a]benzimidazole derivatives against Leishmaniadonovani and Trypanosomacruzi. Eur J Med Chem 84:395-403

7. El-Gohary NS, Shaaban MI (2017) Synthesis and biological evaluation of a new series of benzimidazole derivatives as antimicrobial, antiquorumsensing and antitumor agents. Eur J Med Chem 131:255-262

8. Luo Y, Yao JP, Yang L, Feng CL, Tang W, Wang GF, Zuo JP, Lu W (2011) Synthesis and anti-Hepatitis B virus activity of a novel class of thiazolylbenzimidazole derivatives. Arch Pharm Chem Life Sci 2:78-83

9. Lee-Dutra A, Arienti KL, Buzard DJ, Hack MD, Khatuya H, Desai PJ, Nguyen S, Thurmond RL, Karlsson L, Edwards JP, Breitenbucher JG (2006) Identification of 2-arylbenzimidazoles as potent human histamine $\mathrm{H} 4$ receptor ligands. Bioorg Med Chem Lett 16:6043-6048

10. Sogame S, Suenaga Y, Atobe M, Kawanishi M, Tanaka E, Miyoshi S (2014) Discovery of a benzimidazole series of ADAMTS-5 (aggrecanase-2) inhibitors by scaffold hopping. Eur J Med Chem 71:250-258

11. Shingalapur RV, Hosamani KM, Keri RS (2009) Synthesis and evaluation of in vitro anti-microbial and anti-tubercular activity of 2-styryl benzimidazoles. Eur J Med Chem 44:4244-4248

12. Cinarli A, Gurbuz D, Tavman A, Birteksoz AS (2011) Synthesis, spectral characterizations and antimicrobial activity of some Schiff bases of 4-chloro-2-aminophenol. Bull Chem Soc Ethiop 25:407-417

13. Kumar $P$, Narasimhan B, Yogeswari P, Sriram D (2010) Synthesis and antitubercular activities of substituted benzoic acid $N^{\prime}$-(substituted benzylidene/furan-2-ylmethylene)-N-(pyridine-3-carbonyl)-hydrazides. Eur J Med Chem 45:6085-6089

14. Skehan P, Storeng R, Scudiero D, Monks A, McMahon J, Vistica D, Warren JT, Bokesch H, Kenney S, Boyd MR (1990) New colorimetric cytotoxicity assay for anticancer-drug screening. J Natl Cancer Inst 82:1107-1112

15. Cappucino JG, Sherman N (1999) Microbiology—a laboratory manual. Addison Wesley California 263

16. Pharmacopoeia of India, vol.-I (2007) Controller of publication, ministry of health department, Govt. of India, New Delhi, 37 